\title{
GENERALIZED FRACTIONAL KINETIC EQUATIONS: ANOTHER POINT OF VIEW
}

\author{
DAVID MÁRQUEZ-CARRERAS, ${ }^{*}$ Universitat de Barcelona
}

\begin{abstract}
In this paper we deal with generalized fractional kinetic equations driven by a Gaussian noise, white in time and correlated in space, and where the diffusion operator is the composition of the Bessel and Riesz potentials for any fractional parameters. We give results on the existence and uniqueness of solutions by means of a weak formulation and study the Hölder continuity. Moreover, we prove the existence of a smooth density associated to the solution process and study the asymptotics of this density. Finally, when the diffusion coefficient is constant, we look for its Gaussian index.
\end{abstract}

Keywords: Stochastic fractional kinetic and heat equations; Bessel and Riesz potentials; Gaussian processes; Malliavin calculus

2000 Mathematics Subject Classification: Primary 60G60; 60H15; 60H30

Secondary 60G10; 60G15; 60H07

\section{Introduction}

In this paper we deal with the following kind of equations:

$$
\partial_{t} c(t, x)+(I-\Delta)^{\alpha / 2}(-\Delta)^{\gamma / 2} c(t, x)=f(c(t, x)) \dot{\varepsilon}(t, x),
$$

where $t \in \mathbb{R}_{+}, x \in \mathbb{R}^{d}, c(0, x)=0, \alpha \geq 0, \gamma>0, f$ is a measurable function, and $\dot{\varepsilon}$ is a Gaussian noise. We will specify later the required conditions on the function $f$ and the Gaussian noise $\dot{\varepsilon}$. In (1.1), $I$ and $\Delta$ are the identity and Laplacian operators, respectively, and the operators $(I-\Delta)^{\alpha / 2}$ and $(-\Delta)^{\gamma / 2}$ have to be interpreted as the inverses of the Bessel and Riesz potentials, respectively. These operators are widely dealt with, for instance, in the books of Samko et al. [19] and Stein [21], and more specifically in the paper by Anh et al. [6].

This type of generalized fractional kinetic equation (known also as the fractional diffusion equation or the fractional heat equation) was introduced to model some physical phenomena, such as diffusion in porous media with fractal geometry, kinematics in viscoelastic media, propagation of seismic waves, turbulence, etc. For more information and details about these modelings, we refer the reader to the long list of references in [4]. Nowadays, we can find a lot of applications of these equations in turbulence, ecology, hydrology, geophysics, image analysis, neurophysiology, economics and finance, etc. (see [3] and the references therein). Moreover, there are many examples of equations of the form (1.1), including, for instance, the classical heat stochastic equation for $\alpha=0$ and $\gamma=2$, and the generalized heat equation for $\alpha=0$ and $\gamma>0$.

Recently, several authors have studied this sort of equation and other similar equations from a mathematical point of view. First of all we would like to mention a nice article by

Received 19 September 2007; revision received 22 April 2009.

* Postal address: Departament de Probabilitat, Lògica i Estadística, Facultat de Matemàtiques, Universitat de Barcelona, Gran Via 585, 08007-Barcelona, Spain. Email address: davidmarquez@ub.edu

Partially supported by the grant MTM 2006-01351. 
Angulo et al. [3]. In [3] the authors considered (1.1) with $f=1, \dot{\varepsilon}$ a Gaussian space-time white noise, and bounded and unbounded spatial domains. They connected it with the Eulerian theory of turbulence dispersion by means of the advection-diffusion equation

$$
\partial_{t} c+\nabla \cdot(u c)=\kappa \Delta c, \quad t \in \mathbb{R}_{+}, x \in \mathbb{R}^{d},
$$

where $c(t, x)$ is the concentration field, $\nabla$ is the gradient vector, $u(t, x)$ is the velocity vector field, and $\kappa$ is the molecular diffusivity (they also gave a very interesting connection with the Lagrangian theory). The paper was devoted to the study of some sample path properties of (1.1). In the unbounded case $\left(x \in \mathbb{R}^{d}\right)$, the authors obtained a solution in terms of the Fourier transform of the associated Green function; they checked that at each time $t \in \mathbb{R}_{+}$the solution is a homogeneous random field, calculated its spatial spectral density, and obtained a bound for the variance of the increments; they also observed that the solution is asymptotically stationary in time.

We would also like to mention the papers [2], [4]-[6], [10], and [18]. Anh and Leonenko [5] and Ruiz-Medina et al. [18] studied the following version of the fractional kinetic equation:

$$
\partial_{t} c(t, x)+\tau(I-\Delta)^{\alpha / 2}(-\Delta)^{\gamma / 2} c(t, x)=0, \quad \tau>0 .
$$

Anh and Leonenko [5] considered a measurable random field as an initial condition of (1.2) and presented a renormalization and homogenization theory for this type of equation and more general versions. However, in [18] the initial condition considered was an exponential function of the fractional Riesz-Bessel motion. The papers of Angulo et al. [2] and Anh and Leonenko [4] dealt with a more general type of equation, replacing $\partial_{t}$ by $\partial_{t}^{\beta}$ in (1.1) and (1.2), respectively. Finally, Márquez-Carreras and Florit [10] carried out a detailed study of

$$
\partial_{t} c(t, x)+(-\Delta)^{\gamma / 2} c(t, x)=f(c(t, x)) \dot{\varepsilon}(t, x)+g(c(t, x)) .
$$

On the one hand, this equation is more particular than (1.1) because only $\alpha=0$ is considered, but, on the other hand, it is more general than (1.1) because the term $g$ is added.

In this paper, regarding the structure of (1.1) and comparing with the papers of Angulo, Anh, Leonenko, and others, our study has essentially two different aspects: the function $f$ and the characteristics of our Gaussian noise $\dot{\varepsilon}$. Firstly, we generalize some of the results of Angulo et al. [3] to the nonlinear case. Moreover, we study some new properties for the solution of this class of stochastic partial differential equations (1.1), including, for instance, the Hölder continuity in time and in space and the existence of a smooth density. Secondly, our class of stochastic partial differential equations is driven by a Gaussian noise, white in time and correlated in space (specified in the following section); thanks to this fact, most of the results and properties of this paper do not depend on the dimension $d$ and are true for any positive $\alpha$ and $\gamma$.

The paper is organized as follows. In Section 2 we introduce the Gaussian process and describe via a weak formulation what we mean by a solution of (1.1). We also prove results on the existence and uniqueness of the solution. In Section 3, assuming that $f=1$, we check that spatially the solution of (1.1) is a Gaussian field with zero mean, stationary increments, and a continuous covariance function. We also find its index (see Definition 3.1). Moreover, we also show that the solution is not stationary in time but tends to a stationary process. Assuming again that the function $f$ is not a constant, Section 4 will be devoted to the study of some different properties of the solution of (1.1), such as the Hölder continuity (in time and in space) and the existence of a smooth density. Finally, in Section 5 we study the asymptotic behavior of 
the density in a particular but important point. More specifically, we analyze in this point the asymptotic behavior of the density when we perturb the noise and this perturbation tends to 0 .

As usual, all constants will be denoted by $C$, independently of its value.

\section{A solution by means of a weak formulation}

First of all, we introduce our Gaussian noise. Let $\mathscr{D}\left(\mathbb{R}^{d+1}\right)$ be the space of Schwartz test functions (see, for instance, [20]); then, on a probability space $(\Omega, \mathcal{F}, \mathrm{P})$, the noise

$$
\varepsilon=\left\{\varepsilon(\phi), \phi \in \mathscr{D}\left(\mathbb{R}^{d+1}\right)\right\}
$$

is an $L^{2}(\Omega, \mathcal{F}, \mathrm{P})$-valued centered Gaussian process with covariance functional

$$
\Xi(\phi, \psi)=\int_{\mathbb{R}_{+}} \mathrm{d} s \int_{\mathbb{R}^{d}} \Gamma(\mathrm{d} x)(\phi(s, \cdot) * \tilde{\psi}(s, \cdot))(x),
$$

where $\tilde{\psi}(s, x)=\psi(s,-x)$, and $\Gamma$ is a nonnegative, nonnegative definite, tempered measure, and is therefore symmetric. Denote by $\mu$ the spectral measure of $\Gamma$, which is also a nonnegative tempered measure. Then,

$$
\Xi(\phi, \psi)=\int_{\mathbb{R}_{+}} \mathrm{d} s \int_{\mathbb{R}^{d}} \mu(\mathrm{d} \xi) \mathcal{F} \phi(s, \cdot)(\xi) \overline{\mathcal{F} \psi(s, \cdot)(\xi)},
$$

where $\mathcal{F}$ denotes the Fourier transform and $\bar{z}$ denotes the complex conjugate of $z$. Since the spectral measure $\mu$ is a nontrivial tempered measure, we can ensure that there exist positive constants $k_{1}, k_{2}$, and $K$ such that

$$
k_{1}<\int_{\{|\xi| \leq K\}} \mu(\mathrm{d} \xi)<k_{2} .
$$

As usual, the Gaussian process $\varepsilon$ can be extended to a worthy martingale measure, in the sense given by Walsh [22],

$$
\eta=\left\{\eta_{t}(A), t \in \mathbb{R}_{+}, A \in \mathscr{B}_{b}\left(\mathbb{R}^{d}\right)\right\},
$$

where $\mathcal{B}_{b}\left(\mathbb{R}^{d}\right)$ are the bounded Borel subsets of $\mathbb{R}^{d}$.

Dalang [7] presented an extension of Walsh's stochastic integral that requires the following integrability condition in terms of the Fourier transform of $\Gamma$ :

$$
\int_{0}^{T} \mathrm{~d} t \int_{\mathbb{R}^{d}} \mu(\mathrm{d} \xi)|\mathcal{F} G(t, \cdot)(\xi)|^{2}<\infty,
$$

where $G$ is the fundamental solution of (1.1), that is, the solution of

$$
\partial_{t} G(t, x)+(I-\Delta)^{\alpha / 2}(-\Delta)^{\gamma / 2} G(t, x)=0 .
$$

Provided that (2.2) is satisfied and assuming conditions on $f$ that will be described later, we will understand a solution of (1.1) to be a jointly measurable adapted process $\{c(t, x),(t, x) \in$ $\mathbb{R}_{+} \times \mathbb{R}^{d}$ \} satisfying the integral form

$$
c(t, x)=\int_{0}^{t} \int_{\mathbb{R}^{d}} G(t-s, x-y) f(c(s, y)) \eta(\mathrm{d} s, \mathrm{~d} y),
$$


where the stochastic integral in (2.4) is defined with respect to the $\mathcal{F}_{t}$-martingale measure $\eta_{t}$. For more details, we refer the reader to [7] and [8].

In order to apply these ideas, we need the expression of the Fourier transform of $G$. Anh and Leonenko [4], for example, showed that (2.3) is equivalent to the problem

$$
\partial_{t} \mathcal{F} G(t, \cdot)(\xi)+|\xi|^{\alpha}\left(1+|\xi|^{2}\right)^{\gamma / 2} \mathcal{F} G(t, \cdot)(\xi)=0 .
$$

Using the arguments described in [4] and [9], (2.5) has a unique solution given by

$$
\mathcal{F} G(t, \cdot)(\xi)=\exp \left\{-t|\xi|^{\alpha}\left(1+|\xi|^{2}\right)^{\gamma / 2}\right\} .
$$

So, the fundamental solution of (2.3) can be written as

$$
G(t, x)=\frac{1}{(2 \pi)^{n}} \int_{\mathbb{R}^{d}} \mathrm{e}^{\mathrm{i}\langle x, \xi\rangle} \exp \left\{-t|\xi|^{\alpha}\left(1+|\xi|^{2}\right)^{\gamma / 2}\right\} \mathrm{d} \xi
$$

We now prove (2.2), assuming an integrability condition on the spectral measure.

Lemma 2.1. Assume that the spectral measure $\mu$ associated to $\varepsilon$ satisfies

$$
\int_{\mathbb{R}^{d}} \frac{\mu(\mathrm{d} \xi)}{\left(1+|\xi|^{2}\right)^{(\alpha+\gamma) / 2}}<\infty
$$

Then condition (2.2) is satisfied.

Proof. Fix $K>0$. We have

$$
\int_{0}^{T} \mathrm{~d} t \int_{\mathbb{R}^{d}} \mu(\mathrm{d} \xi)|\mathcal{F} G(t, \cdot)(\xi)|^{2}=: A_{1}+A_{2},
$$

where

$$
\begin{aligned}
& A_{1}=\int_{0}^{T} \mathrm{~d} t \int_{\{|\xi| \leq K\}} \mu(\mathrm{d} \xi)|\mathcal{F} G(t, \cdot)(\xi)|^{2}, \\
& A_{2}=\int_{0}^{T} \mathrm{~d} t \int_{\{|\xi|>K\}} \mu(\mathrm{d} \xi)|\mathcal{F} G(t, \cdot)(\xi)|^{2} .
\end{aligned}
$$

On the one hand, as $|\mathcal{F} G(t, \cdot)(\xi)| \leq 1$, the upper bound in (2.1) implies that

$$
A_{1} \leq T k_{2}<+\infty \text {. }
$$

On the other hand, taking into account (2.6), Fubini's theorem yields

$$
A_{2}=\int_{\{|\xi|>K\}} \frac{1-\exp \left\{-T|\xi|^{\alpha}\left(1+|\xi|^{2}\right)^{\gamma / 2}\right\}}{|\xi|^{\alpha}\left(1+|\xi|^{2}\right)^{\gamma / 2}} \mu(\mathrm{d} \xi)
$$

Since $|\xi|>K$, we have

$$
\frac{1}{|\xi|^{\alpha}\left(1+|\xi|^{2}\right)^{\gamma / 2}} \leq \frac{1}{|\xi|^{\alpha+\gamma}} \leq\left(\frac{1+K^{2}}{K^{2}}\right)^{(\alpha+\gamma) / 2} \frac{1}{\left(1+|\xi|^{2}\right)^{(\alpha+\gamma) / 2}}
$$

Then, as $\mathrm{e}^{-x} \leq 1$ for all $x \geq 0$, thanks to (2.7), we obtain

$$
A_{2}<+\infty \text {. }
$$

We finish the proof of this lemma by putting together (2.8), (2.9), and (2.10). 
In the following proposition we show the existence and uniqueness of the solution in the sense given by (2.4).

Proposition 2.1. Assume that the spectral measure $\mu$ satisfies (2.7) and that the function $f$ is globally Lipschitz. Then, (2.4) has a unique adapted solution and, for any $T>0$ and $p \in[1,+\infty)$,

$$
\sup _{(t, x) \in[0, T] \times \mathbb{R}^{d}} \mathrm{E}\left[|c(t, x)|^{p}\right]<\infty .
$$

Moreover, this unique solution is mean-square continuous.

Proof. The result can be proved using the standard argument based on Picard's iterations. We give a sketch of the proof. Define

$$
\begin{aligned}
& c_{0}(t, x)=0 \\
& c_{n}(t, x)=\int_{0}^{t} \int_{\mathbb{R}^{d}} G(t-s, x-y) f\left(c_{n-1}(s, y)\right) \eta(\mathrm{d} s, \mathrm{~d} y), \quad n \geq 1 .
\end{aligned}
$$

Let $I_{t}^{d}=[0, t] \times \mathbb{R}^{d}$. First we easily prove that $c_{n}(t, x)$ is well defined and then, using Burkholder's inequality, we can show that, for any $n \geq 0$ and $t \in[0, T]$,

$$
\sup _{(s, x) \in I_{t}^{d}} \mathrm{E}\left[\left|c_{n}(s, x)\right|^{2}\right]<\infty,
$$

and, by means of an extension of Gronwall's lemma (see Lemma 15 of [7]), that

$$
\sup _{n \geq 0} \sup _{(t, x) \in I_{T}^{d}} \mathrm{E}\left[\left|c_{n}(t, x)\right|^{2}\right]<\infty .
$$

The same kind of argument allows us to check (2.11) and (2.12), changing the power 2 for $p>2$. Moreover, we can also prove that $\left\{c_{n}(t, x), n \geq 0\right\}$ converges uniformly in $L^{p}$, denoting this limit by $c(t, x)$. We can check that $c(t, x)$ satisfies (2.4). Then it is adapted and

$$
\sup _{(t, x) \in I_{T}^{d}} \mathrm{E}\left[|c(t, x)|^{p}\right]<\infty .
$$

The uniqueness can be accomplished by a similar argument.

The key to the continuity is to show that these Picard iterations are mean-square continuous. Then, it can be easily extended to $c(t, x)$. In order to show the most important ideas of the mean-square continuity, we give some steps of the proof for $\left\{c_{n}(t, x), n \geq 0\right\}$.

As for the time increments, we have, for any $(t, x) \in I_{T}^{d}$ and $u>0$ (satisfying $t+u<T$ ),

$$
\begin{aligned}
& \mathrm{E}\left[\left|c_{n}(t+u, x)-c_{n}(t, x)\right|^{2}\right] \\
& \leq \mathrm{E}\left[\left|\int_{0}^{t} \int_{\mathbb{R}^{d}}[G(t+u-s, x-y)-G(t-s, x-y)] f\left(c_{n-1}(s, y)\right) \eta(\mathrm{d} s, \mathrm{~d} y)\right|^{2}\right] \\
& \quad+\mathrm{E}\left[\left|\int_{t}^{t+u} \int_{\mathbb{R}^{d}} G(t+u-s, x-y) f\left(c_{n-1}(s, y)\right) \eta(\mathrm{d} s, \mathrm{~d} y)\right|^{2}\right] .
\end{aligned}
$$

Using the Lipschitz condition on $f$ and (2.11), we can bound the first term of (2.13) by

$$
C \int_{0}^{t} \mathrm{~d} s \int_{\mathbb{R}^{d}} \mu(\mathrm{d} \xi)|\mathcal{F} G(t+u-s, \cdot)(\xi)-\mathcal{F} G(t-s, \cdot)(\xi)|^{2} .
$$


Then, since

$$
|\mathcal{F} G(t+u-s, \cdot)(\xi)-\mathcal{F} G(t-s, \cdot)(\xi)|^{2} \leq \exp \left\{-2(t-s)|\xi|^{\alpha}\left(1+|\xi|^{2}\right)^{\gamma / 2}\right\}
$$

Lemma 2.1 and the dominated convergence theorem imply that the first term of the right-hand side of (2.13) converges to 0 as $u \searrow 0$. The second term is easier. This proves the right continuity; the left continuity is proved in the same way.

Concerning the spatial increment, we have, for any $(t, x),(t, z) \in I_{T}^{d}$,

$$
\begin{aligned}
& \mathrm{E}\left[\left|c_{n}(t, x)-c_{n}(t, z)\right|^{2}\right] \\
& \quad \leq C \int_{0}^{t} \mathrm{~d} s \int_{\mathbb{R}^{d}} \mu(\mathrm{d} \xi)|\mathcal{F} G(t-s, x-\cdot)(\xi)-\mathcal{F} G(t-s, z-\cdot)(\xi)|^{2} \\
& \quad \leq C \int_{0}^{t} \mathrm{~d} s \int_{\mathbb{R}^{d}} \mu(\mathrm{d} \xi)\left|\mathrm{e}^{\mathrm{i}\langle x-z, \xi\rangle}-1\right|^{2}|\mathcal{F} G(s, z-\cdot)(\xi)|^{2} .
\end{aligned}
$$

Then, thanks to the dominated convergence theorem, we can prove that the right-hand side of the above equation converges to 0 as $|x-z| \rightarrow 0$.

\section{Index- $\lambda$ Gaussian field}

In this section we check that spatially the solution of (2.4) with $f=1$ satisfies the following property defined and studied widely, for instance, in [1].

Definition 3.1. Let $X(\rho)$ be a Gaussian field that has zero mean, stationary increments, and a continuous covariance function. Set $\sigma^{2}(\theta)=\mathrm{E}\left[|X(\rho+\theta)-X(\rho)|^{2}\right]$. Then, if there exists $\lambda \in(0,1]$ such that

$$
\lambda=\sup \left\{\hat{\lambda}: \sigma(\theta)=o\left(\|\theta\|^{\hat{\lambda}}\right),\|\theta\| \downarrow 0\right\},
$$

we call $X$ an index- $\lambda$ Gaussian field.

We will also observe that the solution is not stationary in time but converges to a stationary process as the time tends to $\infty$. In this section we essentially show that our solution (driven by the Gaussian noise defined in Section 2) has some similar properties to the solution studied in [3].

Theorem 3.1. Assume that $f=1$ and that the spectral measure $\mu$ associated to $\varepsilon$ satisfies

$$
\int_{\mathbb{R}^{d}} \frac{\mu(\mathrm{d} \xi)}{\left(1+|\xi|^{2}\right)^{(\alpha+\gamma) q / 2}}<\infty
$$

for some $q \in(0,1)$. Then, for a fixed time $t \in \mathbb{R}_{+}$, the spatial covariance function of the solution of (2.4) with $f=1$ is

$$
R_{t}(x-z)=\int_{\mathbb{R}^{d}} \mathrm{e}^{-\mathrm{i}\langle x-z, \xi\rangle} \frac{1-\exp \left\{-2 t|\xi|^{\alpha}\left(1+|\xi|^{2}\right)^{\gamma / 2}\right\}}{2|\xi|^{\alpha}\left(1+|\xi|^{2}\right)^{\gamma / 2}} \mu(\mathrm{d} \xi) .
$$

Moreover, at each fixed time $t \in \mathbb{R}_{+}$,

- if $\alpha+\gamma \geq 2$ then $c(t, \cdot)$ is an index- $(1-q)$ Gaussian field;

- if $\alpha+\gamma<2$ then $c(t, \cdot)$ is an index- $(1-q)(\alpha+\gamma) / 2$ Gaussian field. 
Proof. We first calculate the spatial covariance for a fixed time $t \in \mathbb{R}_{+}$. By means of the definition of a Fourier transform, a variable change, and Fubini's theorem, we obtain, for any $x, z \in \mathbb{R}^{d}$,

$$
\begin{aligned}
\mathrm{E}[c(t, x) \overline{c(t, z)}] & =\int_{0}^{t} \mathrm{~d} s \int_{\mathbb{R}^{d}} \mu(\mathrm{d} \xi) \mathcal{F} G(t-s, x-\cdot)(\xi) \overline{\mathcal{F} G(t-s, z-\cdot)(\xi)} \\
& =\int_{0}^{t} \mathrm{~d} s \int_{\mathbb{R}^{d}} \mu(\mathrm{d} \xi) \mathrm{e}^{-\mathrm{i}\langle x-z, \xi\rangle}|\mathcal{F} G(t-s, \cdot)(\xi)|^{2} \\
& =\int_{0}^{t} \mathrm{~d} s \int_{\mathbb{R}^{d}} \mu(\mathrm{d} \xi) \mathrm{e}^{-\mathrm{i}\langle x-z, \xi\rangle} \exp \left\{-2(t-s)|\xi|^{\alpha}\left(1+|\xi|^{2}\right)^{\gamma / 2}\right\} \\
& =\int_{\mathbb{R}^{d}} \mathrm{e}^{-\mathrm{i}\langle x-z, \xi\rangle} \frac{1-\exp \left\{-2 t|\xi|^{\alpha}\left(1+|\xi|^{2}\right)^{\gamma / 2}\right\}}{2|\xi|^{\alpha}\left(1+|\xi|^{2}\right)^{\gamma / 2}} \mu(\mathrm{d} \xi) \\
& =R_{t}(x-z) .
\end{aligned}
$$

Moreover, for a fixed time $t \in \mathbb{R}_{+}$, the process $c(t, x)$ is a Gaussian field that has zero mean, stationary increments, and a continuous covariance function.

We now study the index. For $t \in \mathbb{R}_{+}, x \in \mathbb{R}^{d}$, and small $\theta \in \mathbb{R}^{d}$, we have

$$
\begin{aligned}
\sigma^{2}(\theta) & =\mathrm{E}\left[|c(t, x+\theta)-c(t, x)|^{2}\right] \\
& =\mathrm{E}\left[\left|\int_{0}^{t} \int_{\mathbb{R}^{d}}(G(t-s, x+\theta-y)-G(t-s, x-y)) \eta(\mathrm{d} s, \mathrm{~d} y)\right|^{2}\right] \\
& =\int_{0}^{t} \mathrm{~d} s \int_{\mathbb{R}^{d}} \mu(\mathrm{d} \xi)|\mathcal{F} G(t-s, x+\theta-\cdot)(\xi)-\mathcal{F} G(t-s, x-\cdot)(\xi)|^{2} \\
& =: B_{1}+B_{2},
\end{aligned}
$$

where

$$
\begin{aligned}
& B_{1}=\int_{0}^{t} \mathrm{~d} s \int_{\{|\xi|<K\}} \mu(\mathrm{d} \xi)|\mathcal{F} G(t-s, x+\theta-\cdot)(\xi)-\mathcal{F} G(t-s, x-\cdot)(\xi)|^{2} \\
& B_{2}=\int_{0}^{t} \mathrm{~d} s \int_{\{|\xi| \geq K\}} \mu(\mathrm{d} \xi)|\mathcal{F} G(t-s, x+\theta-\cdot)(\xi)-\mathcal{F} G(t-s, x-\cdot)(\xi)|^{2}
\end{aligned}
$$

The first term is easy and can be dealt with in the same way for any value of $\alpha+\gamma$. Indeed, the fact that the Fourier transform of $G$ is bounded by 1 , the mean value theorem, and property (2.1) imply that

$$
\begin{aligned}
B_{1} & =\int_{0}^{t} \mathrm{~d} s \int_{\{|\xi|<K\}} \mu(\mathrm{d} \xi)\left|\mathrm{e}^{-\mathrm{i}\langle x+\theta, \xi\rangle}-\mathrm{e}^{-\mathrm{i}\langle x, \xi\rangle}\right|^{2}|\mathcal{F} G(t-s, \cdot)(\xi)|^{2} \\
& \leq C \int_{0}^{t} \mathrm{~d} s \int_{\{|\xi|<K\}} \mu(\mathrm{d} \xi)|\langle\theta, \xi\rangle|^{2} \\
& \leq C|\theta|^{2}
\end{aligned}
$$

The other term is more difficult. Let $h \in(0,1-q)$. We distinguish two cases depending on the value of $\alpha+\gamma$. We first study when $\alpha+\gamma \geq 2$. Applying the mean value theorem, Fubini's 
theorem, the fact that $1-\mathrm{e}^{-x} \leq 1$ for all $x>0$, and hypothesis (3.1), we have

$$
\begin{aligned}
B_{2} & =\int_{0}^{t} \mathrm{~d} s \int_{\{|\xi| \geq K\}} \mu(\mathrm{d} \xi)\left|\mathrm{e}^{-\mathrm{i}\langle x+\theta, \xi\rangle}-\mathrm{e}^{-\mathrm{i}\langle x, \xi\rangle}\right|^{2}|\mathcal{F} G(t-s, \cdot)(\xi)|^{2} \\
& \leq 4 \int_{0}^{t} \mathrm{~d} s \int_{\{|\xi| \geq K\}} \mu(\mathrm{d} \xi)\left|\frac{1}{2}\left(\mathrm{e}^{-\mathrm{i}\langle x+\theta, \xi\rangle}-\mathrm{e}^{-\mathrm{i}\langle x, \xi\rangle}\right)\right|^{2 h}|\mathcal{F} G(t-s, \cdot)(\xi)|^{2} \\
& \leq C \int_{0}^{t} \mathrm{~d} s \int_{\{|\xi| \geq K\}} \mu(\mathrm{d} \xi)|\theta|^{2 h}|\xi|^{2 h} \exp \left\{-2(t-s)|\xi|^{\alpha}\left(1+|\xi|^{2}\right)^{\gamma / 2}\right\} \\
& \leq C|\theta|^{2 h} \int_{\{|\xi| \geq K\}} \frac{|\xi|^{2 h}}{|\xi|^{\alpha}\left(1+|\xi|^{2}\right)^{\gamma / 2}} \mu(\mathrm{d} \xi) \\
& \leq C|\theta|^{2 h} \int_{\{|\xi| \geq K\}} \frac{1}{\left(1+|\xi|^{2}\right)^{(\alpha+\gamma-2 h) / 2}} \mu(\mathrm{d} \xi) \\
& \leq C|\theta|^{2 h} \int_{\{|\xi| \geq K\}} \frac{1}{\left(1+|\xi|^{2}\right)^{(1-h)(\alpha+\gamma) / 2}} \mu(\mathrm{d} \xi) \\
& \leq C|\theta|^{2 h} .
\end{aligned}
$$

We now assume that $\alpha+\gamma<2$. Then, similar arguments yield

$$
\begin{aligned}
B_{2} & =\int_{0}^{t} \mathrm{~d} s \int_{\{|\xi| \geq K\}} \mu(\mathrm{d} \xi)\left|\mathrm{e}^{-\mathrm{i}\langle x+\theta, \xi\rangle}-\mathrm{e}^{-\mathrm{i}\langle x, \xi\rangle}\right|^{2}|\mathcal{F} G(t-s, \cdot)(\xi)|^{2} \\
& \leq 4 \int_{0}^{t} \mathrm{~d} s \int_{\{|\xi| \geq K\}} \mu(\mathrm{d} \xi)\left|\frac{1}{2}\left(\mathrm{e}^{-\mathrm{i}\langle x+\theta, \xi\rangle}-\mathrm{e}^{-\mathrm{i}\langle x, \xi\rangle}\right)\right|^{h(\alpha+\gamma)}|\mathcal{F} G(t-s, \cdot)(\xi)|^{2} \\
& \leq C|\theta|^{h(\alpha+\gamma)} \int_{\{|\xi| \geq K\}} \frac{|\xi|^{h(\alpha+\gamma)}}{|\xi|^{\alpha}\left(1+|\xi|^{2}\right)^{\gamma / 2}} \mu(\mathrm{d} \xi) \\
& \leq C|\theta|^{h(\alpha+\gamma)} \int_{\{|\xi| \geq K\}} \frac{1}{\left(1+|\xi|^{2}\right)^{(1-h)(\alpha+\gamma) / 2}} \mu(\mathrm{d} \xi) \\
& \leq C|\theta|^{h(\alpha+\gamma)}
\end{aligned}
$$

As in [3], process (2.4) with $f=1$ is not stationary in time but, as $t$ tends to $\infty$, it converges to a stationary process. This means that the limiting-time process is stationary in time and space.

Proposition 3.1. Assume that $f=1$ and that the spectral measure $\mu$ associated to $\varepsilon$ satisfies (3.1) for some $q \in(0,1)$. Then, for $t \in \mathbb{R}_{+}, \tau \in \mathbb{R}$ such that $t+\tau \in \mathbb{R}_{+}$, and $x, z \in \mathbb{R}^{d}$, the asymptotic homogeneous spatio-temporal covariance function of $c(t+\tau, x)$ and $c(t, z)$ is

$$
R(\tau, x-z)=\int_{\mathbb{R}^{d}} \mathrm{e}^{-\mathrm{i}\langle x-z, \xi\rangle} \frac{\exp \left\{-|\tau||\xi|^{\alpha}\left(1+|\xi|^{2}\right)^{\gamma / 2}\right\}}{2|\xi|^{\alpha}\left(1+|\xi|^{2}\right)^{\gamma / 2}} \mu(\mathrm{d} \xi)
$$

Moreover, $c(\cdot, x)$ is asymptotically in time an index- $(1-q) / 2$ Gaussian field. 
Proof. For $t, \tau \in \mathbb{R}_{+}$(for $\tau \in \mathbb{R}_{-}$such that $t+\tau \in \mathbb{R}_{+}$, we argue similarly), and $x, z \in \mathbb{R}^{d}$, we have

$$
\begin{aligned}
\mathrm{E}[c(t+\tau, x) \overline{c(t, z)}]= & \int_{0}^{t} \mathrm{~d} s \int_{\mathbb{R}^{d}} \mu(\mathrm{d} \xi) \mathcal{F} G(t+\tau-s, x-\cdot)(\xi) \overline{\mathcal{F} G(t-s, z-\cdot)(\xi)} \\
= & \int_{0}^{t} \mathrm{~d} s \int_{\mathbb{R}^{d}} \mu(\mathrm{d} \xi) \mathrm{e}^{-\mathrm{i}\langle x-z, \xi\rangle} \mathcal{F} G(t+\tau-s, \cdot)(\xi) \mathcal{F} G(t-s, \cdot)(\xi) \\
= & \int_{0}^{t} \mathrm{~d} s \int_{\mathbb{R}^{d}} \mu(\mathrm{d} \xi) \mathrm{e}^{-\mathrm{i}\langle x-z, \xi\rangle} \exp \left\{-[2(t-s)+\tau]|\xi|^{\alpha}\left(1+|\xi|^{2}\right)^{\gamma / 2}\right\} \\
= & \int_{\mathbb{R}^{d}} \mathrm{e}^{-\mathrm{i}\langle x-z, \xi\rangle} \frac{\exp \left\{-\tau|\xi|^{\alpha}\left(1+|\xi|^{2}\right)^{\gamma / 2}\right\}}{2|\xi|^{\alpha}\left(1+|\xi|^{2}\right)^{\gamma / 2}} \mu(\mathrm{d} \xi) \\
& +\int_{\mathbb{R}^{d}} \mathrm{e}^{-\mathrm{i}\langle x-z, \xi\rangle} \frac{\exp \left\{-(2 t+\tau)|\xi|^{\alpha}\left(1+|\xi|^{2}\right)^{\gamma / 2}\right\}}{2|\xi|^{\alpha}\left(1+|\xi|^{2}\right)^{\gamma / 2}} \mu(\mathrm{d} \xi) \\
= & R_{t}(\tau, x-z) .
\end{aligned}
$$

Moreover, as $t \rightarrow+\infty$, we obtain

$$
R(\tau, x-z)=\lim _{t \rightarrow+\infty} R_{t}(\tau, x-z)=\int_{\mathbb{R}^{d}} \mathrm{e}^{-\mathrm{i}\langle x-z, \xi\rangle} \frac{\exp \left\{-|\tau||\xi|^{\alpha}\left(1+|\xi|^{2}\right)^{\gamma / 2}\right\}}{2|\xi|^{\alpha}\left(1+|\xi|^{2}\right)^{\gamma / 2}} \mu(\mathrm{d} \xi) .
$$

We now tackle the second part of this proposition. We assume that $x \in \mathbb{R}^{d}, t \in \mathbb{R}_{+}$and $\tau \in \mathbb{R}_{+}$are small (the negative case is similar). Then, we have

$$
\mathrm{E}\left[|c(t+\tau, x)-c(t, x)|^{2}\right] \leq K\left(H_{1}+H_{2}\right),
$$

where

$$
\begin{aligned}
H_{1} & =\mathrm{E}\left[\left|\int_{t}^{t+\tau} \int_{\mathbb{R}^{d}} G(t+\tau-s, x-y) \eta(\mathrm{d} s, \mathrm{~d} y)\right|^{2}\right] \\
& =\int_{t}^{t+\tau} \mathrm{d} s \int_{\mathbb{R}^{d}} \mu(\mathrm{d} \xi)|\mathcal{F} G(t+\tau-s, \cdot)(\xi)|^{2} \\
& =: H_{1,1}+H_{1,2}, \\
H_{2} & =\mathrm{E}\left[\left|\int_{0}^{t} \int_{\mathbb{R}^{d}}(G(t+\tau-s, x-y)-G(t-s, x-y)) \eta(\mathrm{d} s, \mathrm{~d} y)\right|^{2}\right] \\
& =\int_{0}^{t} \mathrm{~d} s \int_{\mathbb{R}^{d}} \mu(\mathrm{d} \xi)|\mathcal{F} G(t+\tau-s, \cdot)(\xi)-\mathcal{F} G(t-s, \cdot)(\xi)|^{2} \\
& =: H_{2,1}+H_{2,2},
\end{aligned}
$$

and

$$
\begin{gathered}
H_{1,1}=\int_{t}^{t+\tau} \mathrm{d} s \int_{\{|\xi|<K\}} \mu(\mathrm{d} \xi) \exp \left\{-2(t+\tau-s)|\xi|^{\alpha}\left(1+|\xi|^{2}\right)^{\gamma / 2}\right\} \\
H_{1,2}=\int_{t}^{t+\tau} \mathrm{d} s \int_{\{|\xi| \geq K\}} \mu(\mathrm{d} \xi) \exp \left\{-2(t+\tau-s)|\xi|^{\alpha}\left(1+|\xi|^{2}\right)^{\gamma / 2}\right\} \\
H_{2,1}=\int_{0}^{t} \mathrm{~d} s \int_{\{|\xi|<K\}} \mu(\mathrm{d} \xi) \exp \left\{-2(t-s)|\xi|^{\alpha}\left(1+|\xi|^{2}\right)^{\gamma / 2}\right\} \\
\times\left(1-\exp \left\{-\tau|\xi|^{\alpha}\left(1+|\xi|^{2}\right)^{\gamma / 2}\right\}\right)^{2}
\end{gathered}
$$




$$
\begin{gathered}
H_{2,2}=\int_{0}^{t} \mathrm{~d} s \int_{\{|\xi| \geq K\}} \mu(\mathrm{d} \xi) \exp \left\{-2(t-s)|\xi|^{\alpha}\left(1+|\xi|^{2}\right)^{\gamma / 2}\right\} \\
\times\left(1-\exp \left\{-\tau|\xi|^{\alpha}\left(1+|\xi|^{2}\right)^{\gamma / 2}\right\}\right)^{2}
\end{gathered}
$$

By means of (2.1) we can easily prove that

$$
H_{1,1}+H_{2,1} \leq C\left(|\tau|+|\tau|^{2}\right) .
$$

Let $h \in(0,(1-q) / 2)$. For $H_{1,2}$, Fubini's theorem, the facts that $1-\mathrm{e}^{-x} \leq 1$ and $1-\mathrm{e}^{-x} \leq x$ for all $x>0$, and hypothesis (3.1) imply that

$$
\begin{aligned}
H_{1,2} & \leq C \int_{\{|\xi| \geq K\}} \frac{1-\exp \left\{-2 \tau|\xi|^{\alpha}\left(1+|\xi|^{2}\right)^{\gamma / 2}\right\}}{|\xi|^{\alpha}\left(1+|\xi|^{2}\right)^{\gamma / 2}} \mu(\mathrm{d} \xi) \\
& \leq C \int_{\{|\xi| \geq K\}} \frac{\left(1-\exp \left\{-2 \tau|\xi|^{\alpha}\left(1+|\xi|^{2}\right)^{\gamma / 2}\right\}\right)^{2 h}}{|\xi|^{\alpha}\left(1+|\xi|^{2}\right)^{\gamma / 2}} \mu(\mathrm{d} \xi) \\
& \leq C|\tau|^{2 h} \int_{\{|\xi| \geq K\}} \frac{\mu(\mathrm{d} \xi)}{\left(1+|\xi|^{2}\right)^{(1-2 h)(\alpha+\gamma) / 2}} \\
& \leq C|\tau|^{2 h} .
\end{aligned}
$$

Similarly, we obtain

$$
H_{2,2} \leq C \int_{\{|\xi| \geq K\}} \frac{\left(1-\exp \left\{-\tau|\xi|^{\alpha}\left(1+|\xi|^{2}\right)^{\gamma / 2}\right\}\right)^{2 h}}{|\xi|^{\alpha}\left(1+|\xi|^{2}\right)^{\gamma / 2}} \mu(\mathrm{d} \xi) \leq C|\tau|^{2 h} .
$$

We complete the proof of this second part by putting together (3.2)-(3.7).

\section{Some properties of the solution}

In this section, assuming again that $f$ is a general function, we will prove two properties about $c(t, x)$, the solution to (2.4): the Hölder continuity and the existence of a smooth density.

First of all we state that the solution $c(t, x)$ is Hölder continuous in time and in space, given the Hölder coefficients.

Proposition 4.1. Assume that the spectral measure $\mu$ associated to $\varepsilon$ satisfies (3.1) for some $q \in(0,1)$ and that $f$ is globally Lipschitz. Then, for every $s, t \in[0, T], T>0, x, z \in \mathbb{R}^{d}$, $p \geq 2, \beta_{1} \in(0,(1-q) / 2)$, and $\beta_{2} \in(0,1-q)$, we have

$$
\begin{gathered}
\mathrm{E}\left[|c(t, x)-c(s, x)|^{p}\right] \leq C|t-s|^{\beta_{1} p}, \\
\mathrm{E}\left[|c(t, z)-c(t, x)|^{p}\right] \leq \begin{cases}C|z-x|^{\beta_{2} p} & \text { if } \alpha+\gamma \geq 2, \\
C|z-x|^{\beta_{2} p(\alpha+\gamma) / 2} & \text { if } \alpha+\gamma<2 .\end{cases}
\end{gathered}
$$

Proof. We omit it since the steps of this proof are similar to the arguments given in Theorem 3.1 and Proposition 3.1.

We now tackle the existence of a smooth density.

Theorem 4.1. Assume that $f$ is $\mathrm{C}^{\infty}$ with bounded derivatives of any order and that there exists $f_{0}>0$ such that $|f(y)| \geq f_{0}$ for any $y \in \mathbb{R}$. We also assume that the spectral measure $\mu$ associated to $\varepsilon$ satisfies (3.1) for some $q \in\left(0, \frac{1}{2}\right)$. Then the law of $c(t, x)$, the solution to (2.4), is absolutely continuous with respect to Lebesgue's measure on $\mathbb{R}$ and its density is infinitely differentiable. 
Proof. In order to prove this theorem we need to check that, for fixed $t>0$ and $x \in \mathbb{R}^{d}$, there exist $\rho_{1}, \rho_{2}>0$ such that $\rho_{2}<\rho_{1}<2 \rho_{2}$, positive constants $C_{1}$ and $C_{2}$, and $r_{0} \in[0, T]$ such that, for all $r \in\left[0, r_{0}\right]$,

$$
C_{1} r^{\rho_{1}} \leq \Theta(r):=\int_{0}^{r} \mathrm{~d} t \int_{\mathbb{R}^{d}} \mu(\mathrm{d} \xi)|\mathcal{F} G(s, \cdot)(\xi)|^{2} \leq C_{2} r^{\rho_{2}} .
$$

This result was proved in [15].

We first deal with the lower bound. Using the fact that

$$
\frac{1}{x}\left(1-\mathrm{e}^{-x}\right) \geq \frac{1}{1+x} \text { for all } x>0,
$$

and the property of the spectral measure (2.1), we find that there exists a $K>0$ such that

$$
\begin{aligned}
\Theta(r) & =\int_{0}^{r} \mathrm{~d} t \int_{\mathbb{R}^{d}} \mu(\mathrm{d} \xi) \exp \left\{-2 s|\xi|^{\alpha}\left(1+|\xi|^{2}\right)^{\gamma / 2}\right\} \\
& \geq \int_{\mathbb{R}^{d}} \frac{1-\exp \left\{-2 r|\xi|^{\alpha}\left(1+|\xi|^{2}\right)^{\gamma / 2}\right\}}{2|\xi|^{\alpha}\left(1+|\xi|^{2}\right)^{\gamma / 2}} \mu(\mathrm{d} \xi) \\
& \geq r \int_{\mathbb{R}^{d}} \frac{1}{1+2 r|\xi|^{\alpha}\left(1+|\xi|^{2}\right)^{\gamma / 2}} \mu(\mathrm{d} \xi) \\
& \geq r \int_{\{|\xi|<K\}} \frac{1}{1+2 r|\xi|^{\alpha}\left(1+|\xi|^{2}\right)^{\gamma / 2}} \mu(\mathrm{d} \xi) \\
& \geq \frac{r}{1+2 r_{0} K^{\alpha}\left(1+K^{2}\right)^{\gamma / 2}} \int_{\{|\xi|<K\}} \mu(\mathrm{d} \xi) \\
& \geq C_{1} r .
\end{aligned}
$$

For the proof of the upper bound, we decompose $\Theta(r)$ in (4.1) as follows:

$$
\Theta(r)=\Theta_{1}(r)+\Theta_{2}(r),
$$

where

$$
\begin{aligned}
& \Theta_{1}(r)=\int_{0}^{r} \mathrm{~d} t \int_{\{|\xi|<K\}} \mu(\mathrm{d} \xi) \exp \left\{-2 s|\xi|^{\alpha}\left(1+|\xi|^{2}\right)^{\gamma / 2}\right\}, \\
& \Theta_{2}(r)=\int_{0}^{r} \mathrm{~d} t \int_{\{|\xi| \geq K\}} \mu(\mathrm{d} \xi) \exp \left\{-2 s|\xi|^{\alpha}\left(1+|\xi|^{2}\right)^{\gamma / 2}\right\} .
\end{aligned}
$$

Since $\mathrm{e}^{-x} \leq 1$ for all $x>0$, it is obvious that

$$
\Theta_{1}(r) \leq C r .
$$

On the other hand, using the facts that $1-\mathrm{e}^{-x} \leq 1$ and $1-\mathrm{e}^{-x} \leq x$ for all $x>0$, and hypothesis (3.1), we obtain, for $h=1-q$,

$$
\begin{aligned}
\Theta_{2}(r) & \leq \int_{\{|\xi| \geq K\}} \frac{\left(1-\exp \left\{-2 r|\xi|^{\alpha}\left(1+|\xi|^{2}\right)^{\gamma / 2}\right\}\right)^{h}}{2|\xi|^{\alpha}\left(1+|\xi|^{2}\right)^{\gamma / 2}} \mu(\mathrm{d} \xi) \\
& \leq C r^{h} \int_{\{|\xi| \geq K\}} \frac{\mu(\mathrm{d} \xi)}{\left(1+|\xi|^{2}\right)^{(1-h)(\alpha+\gamma) / 2}} \\
& \leq C r^{h}
\end{aligned}
$$

So, (4.2)-(4.5) imply (4.1). 


\section{Asymptotics of the density}

Consider the evolution integral equation

$$
c^{\varrho}(t, x)=\varrho \int_{0}^{t} \int_{\mathbb{R}^{d}} G(t-s, x-y) f\left(c^{\varrho}(s, y)\right) \eta(\mathrm{d} s, \mathrm{~d} y),
$$

with $\varrho>0$, under the same considerations as in (2.4). In the sequel we assume that $f$ is $\mathcal{C}^{\infty}$ with bounded derivatives of any order and that there exists an $f_{0}>0$ such that $|f(y)| \geq f_{0}$ for any $y \in \mathbb{R}$. We also suppose that the spectral measure $\mu$ associated to $\varepsilon$ satisfies (3.1) for some $q \in\left(0, \frac{1}{2}\right)$. The main theorem of this section will need these assumptions; however, some previous and technical results of this section can be proved with weaker hypotheses.

As a consequence of Theorem 4.1, for $t \in(0, T], x \in \mathbb{R}^{d}$, and $\varrho>0$, we can ensure the existence of a smooth density associated to $c^{\varrho}(t, x)$, which will be denoted by $p_{t, x}^{\varrho}(y)$. As $\varrho \rightarrow 0$, owing to the convergence of the solution of (5.1) to 0 , we expect that the density $p_{t, x}^{\varrho}(y)$ explodes and tends to a degenerate density with all mass in the point $y=0$. In this section we study the Taylor expansion of $p_{t, x}^{\varrho}(y)$ at $\varrho=0$ for $y=0$.

In order to find this Taylor expansion, we will need to check that the process $c^{\varrho}(t, x)$ is smooth with respect to $\varrho$ and differentiable in the Malliavin sense, and that the Malliavin matrix is invertible.

\subsection{Regularity with respect to $\varrho$}

The process $\left\{c^{\varrho}(t, x), \varrho \in(0,1)\right\}$ has a $\mathcal{C}^{\infty}$ version with respect to $\varrho$ and the derivatives $\partial_{\varrho}^{j} c^{\varrho}(t, x)$, denoted by $c_{j}^{\varrho}(t, x)$, satisfy

$$
c_{1}^{\varrho}(t, x)=\int_{0}^{t} \int_{\mathbb{R}^{d}} G(t-s, x-y)\left[f\left(c^{\varrho}(s, y)\right)+\varrho f^{\prime}\left(c^{\varrho}(s, y)\right) c_{1}^{\varrho}(s, y)\right] \eta(\mathrm{d} s, \mathrm{~d} y),
$$

and, for $j \geq 2$,

$$
c_{j}^{\varrho}(t, x)=\Lambda_{j-1}^{\varrho}(t, x)+\varrho \int_{0}^{t} \int_{\mathbb{R}^{d}} G(t-s, x-y) f^{\prime}\left(c^{\varrho}(s, y)\right) c_{j}^{\varrho}(s, y) \eta(\mathrm{d} s, \mathrm{~d} y),
$$

where, if we use the shorthand $\sum^{(i, \ell)}$ for $\sum_{k=i}^{\ell} \sum_{\beta_{1}+\cdots+\beta_{k}=\ell, \beta_{1}, \ldots, \beta_{k} \geq 1}$,

$$
\begin{aligned}
\Lambda_{j-1}^{\varrho}(t, x)=\int_{0}^{t} \int_{\mathbb{R}^{d}} G(t-s, x-y) & {\left[\sum^{(1, j-1)} k_{\beta_{1}, \ldots, \beta_{k}}^{j-1} f^{(k)}\left(c^{\varrho}(s, y)\right) \prod_{n=1}^{k} c_{\beta_{n}}^{\varrho}(s, y) \eta(\mathrm{d} s, \mathrm{~d} y)\right.} \\
& \left.+\varrho \sum^{(2, j)} K_{\beta_{1}, \ldots, \beta_{k}}^{j} f^{(k)}\left(c^{\varrho}(s, y)\right) \prod_{n=1}^{k} c_{\beta_{n}}^{\varrho}(s, y) \eta(\mathrm{d} s, \mathrm{~d} y)\right]
\end{aligned}
$$

and $k_{\beta_{1}, \ldots, \beta_{k}}^{j}$ and $K_{\beta_{1}, \ldots, \beta_{k}}^{j}$ can be computed by induction. We remark that the derivatives of $c^{\varrho}(s, y)$ that appear in $\Lambda_{j-1}^{\varrho}(t, x)$ are of order $\beta_{n}<j$.

Moreover, for $j \geq 1$,

$$
\lim _{\varrho \searrow 0} c_{j}^{\varrho}(t, x)=c_{j}^{0}(t, x) \quad \text { almost surely (a.s.), }
$$

where $c_{j}^{0}(t, x)$ are the solutions of (5.2) and (5.3) replacing $\varrho$ by 0 . In particular,

$$
c_{1}^{0}(t, x)=f(0) \int_{0}^{t} \int_{\mathbb{R}^{d}} G(t-s, x-y) \eta(\mathrm{d} s, \mathrm{~d} y) .
$$


In the sequel we give some ideas of the proof of this regularity. This result is based on the idea of the derivative and the steps given in [11].

For $\varrho \in[0,1]$ and $\delta$ such that $\varrho+\delta \in[0,1]$, we have

$$
c^{\varrho+\delta}(t, x)-c^{\varrho}(t, x)=\delta \Phi_{1}^{\varrho, \delta}(t, x)+\varrho \Phi_{2}^{\varrho, \delta}(t, x),
$$

where

$$
\begin{aligned}
& \Phi_{1}^{\varrho, \delta}(t, x)=\int_{0}^{t} \int_{\mathbb{R}^{d}} G(t-s, x-y) f\left(c^{\varrho+\delta}(s, y)\right) \eta(\mathrm{d} s, \mathrm{~d} y), \\
& \Phi_{2}^{\varrho, \delta}(t, x)=\int_{0}^{t} \int_{\mathbb{R}^{d}} G(t-s, x-y)\left[f\left(c^{\varrho+\delta}(s, y)\right)-f\left(c^{\varrho}(s, y)\right)\right] \eta(\mathrm{d} s, \mathrm{~d} y) .
\end{aligned}
$$

Burkholder's inequality, Hölder's inequality, and Gronwall's lemma easily yield

$$
\sup _{0 \leq t \leq T} \sup _{x \in \mathbb{R}^{d}} \mathrm{E}\left[\left|c^{\varrho+\delta}(t, x)-c^{\varrho}(t, x)\right|^{p}\right] \leq C|\delta|^{p}, \quad p \geq 1,
$$

and Kolmogorov's theorem implies the existence of a continuous version of $\left\{c^{\varrho}(t, x)\right.$, $\varrho \in[0,1]\}$ with $\lim _{\varrho \downarrow 0} c^{\varrho}(t, x)=0$ a.s.

We now show some steps of the differentiability of order $j=1$. We can generalize all the following results by induction on $j$. For any $\varrho \in[0,1]$ and $\delta \in \mathbb{R}-\{0\}$ such that $0 \leq \varrho+\delta \leq 1$, set

$$
\Psi_{\varrho, \delta}(t, x)=\frac{c^{\varrho+\delta}(t, x)-c^{\varrho}(t, x)}{\delta} .
$$

The mean value theorem gives

$\Psi_{\varrho, \delta}(t, x)=\Phi_{1}^{\varrho, \delta}(t, x)+\varrho \int_{0}^{t} \int_{\mathbb{R}^{d}} G(t-s, x-y)\left(\int_{0}^{1} f^{\prime}\left(U_{\varrho, \delta}^{\lambda}(s, y)\right) \mathrm{d} \lambda\right) \Psi_{\varrho, \delta}(s, y) \eta(\mathrm{d} s, \mathrm{~d} y)$, where $U_{\varrho, \delta}^{\lambda}(s, y)=c^{\varrho}(s, y)+\lambda\left(c^{\varrho+\delta}(s, y)-c^{\varrho}(s, y)\right)$.

Using the same arguments as in (5.5), we obtain, for any $p \in[1, \infty)$ and some positive constant $C$,

$$
\sup _{\varrho, \delta} \sup _{(t, x) \in[0, T] \times \mathbb{R}^{d}} \mathrm{E}\left[\left|\Psi_{\varrho, \delta}(t, x)\right|^{p}\right] \leq C,
$$

where the first supremum is over $\varrho \in[0,1], \delta \neq 0,0 \leq \varrho+\delta \leq 1$, and

$$
\sup _{0 \leq t \leq T} \sup _{x \in \mathbb{R}^{d}} \mathrm{E}\left[\left|\Psi_{\varrho, \delta}(t, x)-\Psi_{\varrho^{\prime}, \delta^{\prime}}(t, x)\right|^{p}\right] \leq C\left(\left|\varrho-\varrho^{\prime}\right|^{p}+\left|\delta-\delta^{\prime}\right|^{p}\right) .
$$

Kolmogorov's theorem again implies that $c^{\varrho}(t, x)$ has a differentiable version. We can easily check that

$$
\lim _{\delta \downarrow 0} \mathrm{E}\left[\left|\Psi_{\varrho, \delta}(t, x)-c_{1}^{\varrho}(t, x)\right|^{2}\right]=0
$$

and $\partial_{\varrho} c^{\varrho}(t, x)=c_{1}^{\varrho}(t, x)$ a.s. We can argue as before to show that $\left\{c_{1}^{\varrho}(t, x), \varrho \in[0,1]\right\}$ has a continuous version and $\lim _{\varrho \downarrow} c_{1}^{\varrho}(t, x)=c_{1}^{0}(t, x)$ a.s.

Finally, we have

$$
\hat{c}^{\varrho}(t, x):=\frac{c^{\varrho}(t, x)}{\varrho}=\frac{1}{j+1}\left(c_{j+1}^{0}(t, x)+\varrho \int_{0}^{1}\left(1-\tau^{j+1}\right) c_{j+2}^{\varrho \tau}(t, x) \mathrm{d} \tau\right), \quad 0<\varrho \leq 1,
$$


where $\partial_{\varrho}^{j} \hat{c}^{\varrho}(t, x)=\hat{c}_{j}^{\varrho}(t, x)$. The second equality can be proved following the steps of [13]. Then, for any $j \geq 1$,

$$
\hat{c}_{j}^{0}(t, x):=\lim _{\varrho \downarrow 0} \hat{c}_{j}^{\varrho}(t, x)=\frac{1}{j+1} c_{j+1}^{0}(t, x) \quad \text { a.s. }
$$

This new process will play a very important role in the main proof of this section.

\subsection{Malliavin's derivatives}

Let $\varepsilon$ be the space of measurable functions $\varphi: \mathbb{R}^{d} \rightarrow \mathbb{R}$ such that

$$
\int_{\mathbb{R}^{d}} \Gamma(\mathrm{d} x)[\varphi(\cdot) * \tilde{\psi}(\cdot)](x)<\infty
$$

where $\tilde{\psi}(x)=\psi(-x)$. This space is endowed with the inner product

$$
\langle\varphi, \psi\rangle_{\mathcal{E}}=\int_{\mathbb{R}^{d}} \Gamma(\mathrm{d} x)[\varphi(\cdot) * \tilde{\psi}(\cdot)](x)=\int_{\mathbb{R}^{d}} \Gamma(\mathrm{d} x) \int_{\mathbb{R}^{d}} \mathrm{~d} y \varphi(x-y) \psi(-y) .
$$

Let $\mathscr{H}$ be the completion of $\mathscr{E}$ and $\mathscr{H}_{T}=L^{2}([0, T] ; \mathscr{H})$. This last space is a real separable Hilbert space isomorphic to the reproducing kernel Hilbert space of the centered Gaussian noise (i.e. if $\left.\varphi, \psi \in \mathscr{D}\left([0, T] \times \mathbb{R}^{d}\right), \mathrm{E}[\eta(\varphi) \eta(\psi)]=\langle\varphi, \psi\rangle_{\mathscr{H}_{T}}\right)$. This Gaussian noise $\eta$ can be identified with a Gaussian process $\left\{W(h), h \in \mathscr{H}_{T}\right\}$ as follows. Let $\left\{e_{j}, j \geq 0\right\} \subset \mathcal{E}$ be a CONS of the Hilbert space $\mathscr{H}$. Then

$$
W_{j}(t)=\int_{0}^{t} \int_{\mathbb{R}^{d}} e_{j}(x) \eta(\mathrm{d} s, \mathrm{~d} x), \quad j \in \mathbb{N}, t \in[0, T],
$$

is a sequence of independent standard Brownian motions such that

$$
\eta(\varphi)=\sum_{j=0}^{\infty} \int_{0}^{T}\left\langle\varphi(s, \cdot) e_{j}(\cdot)\right\rangle_{\mathscr{H}} \mathrm{d} W_{j}(s), \quad \varphi \in \mathbb{D}\left([0, T] \times \mathbb{R}^{d}\right),
$$

and, for $h \in \mathscr{H}_{T}$,

$$
W(h)=\sum_{j=0}^{\infty} \int_{0}^{T}\left\langle h(s, \cdot), e_{j}(\cdot)\right\rangle_{\mathscr{H}} \mathrm{d} W_{j}(s) .
$$

As in [17], the Sobolev spaces $\mathbb{D}^{k, p}$ are defined by means of iterations of the derivative operator $D$. For a random variable $X, D^{k} X$ defines a $\mathscr{H}_{T}^{\otimes k}$-valued random variable whenever it exists. For $h \in \mathscr{H}_{T}$, set $D_{h} X=\langle D X, h\rangle_{\mathscr{H}_{T}}$ and, for $r \in[0, T], D_{r} X$ defines an element of $\mathcal{H}$, which is denoted by $D_{r} . X$. Then, for any $h \in \mathcal{H}_{T}$,

$$
D_{h} X=\int_{0}^{T}\left\langle D_{r,} X, h(r)\right\rangle_{\mathscr{H}} \mathrm{d} r
$$

and we write $D_{r, \varphi} X=\left\langle D_{r,}, X, \varphi\right\rangle_{\mathscr{H}}$ for $\varphi \in \mathscr{H}$.

It is not difficult to show as in [14] that, for any $t \geq 0, x \in \mathbb{R}^{d}$, and $\varrho \in[0,1]$, the process $c^{\varrho}(t, x)$ belongs to $\mathbb{D}^{\infty}=\bigcap_{p \geq 1} \bigcap_{k \geq 1} \mathbb{D}^{k, p}(\mathbb{R})$ and that, for any $k \geq 1$ and $p \in[1, \infty)$,

$$
\sup _{\varrho \in[0,1]}\left\|c^{\varrho}(t, x)\right\|_{k, p}<\infty
$$


where $\|\cdot\|_{k, p}$ denotes the norm of the Sobolev space $\mathbb{D}^{k, p}$. Moreover, for any $\varphi \in \mathcal{H}$, the derivatives satisfy, if $r \in[0, t]$,

$$
\begin{aligned}
D_{r, \varphi} c^{\varrho}(t, x)= & \varrho\left\langle G(t-r, x-\cdot) f\left(c^{\varrho}(r, \cdot), \varphi\right\rangle_{\mathscr{H}}\right. \\
& +\varrho \int_{r}^{t} \int_{\mathbb{R}^{d}} G(t-r, x-y) D_{r, \varphi} c^{\varrho}(s, y) f^{\prime}\left(c^{\varrho}(s, y)\right) \eta(\mathrm{d} s, \mathrm{~d} y),
\end{aligned}
$$

and $D_{r, \varphi} c^{\varrho}(t, x)=0$, if $r>t$.

In order to study the Malliavin derivatives, we use the notation of [12]. Let $k \in \mathbb{N}, A_{k}=$ $\left\{\sigma_{i}=\left(r_{i}, \varphi_{i}\right) \in \mathbb{R}_{+} \times \mathcal{H}, i=1, \ldots, k\right\}, \bigvee_{i} r_{i}=r_{1} \vee \cdots \vee r_{k}, \sigma=\left(\sigma_{1}, \ldots, \sigma_{k}\right)$, and $\hat{\sigma}_{i}=\left(\sigma_{1}, \ldots, \sigma_{i-1}, \sigma_{i+1}, \ldots, \sigma_{k}\right)$. Let $\mathbb{P}_{m}$ be the set of $m$ disjoint subsets $p_{1}, \ldots, p_{m}, m=$ $1, \ldots, k$, which are partitions of $A_{k}$, and denote by $\left|p_{i}\right|$ the cardinal of $p_{i}$.

For $X$ belonging to $\mathbb{D}^{k, p}, k \geq 1, p \geq 2$, and a smooth function $g$, the rules of Malliavin's derivatives yield

$$
D_{\sigma}^{k} g(X)=\sum_{m=1}^{k} \sum_{\mathbb{P}_{m}} c_{m} g^{(m)}(X) \prod_{i=1}^{m} D_{p_{i}}^{\left|p_{i}\right|} X
$$

with positive coefficients $c_{m}, m \geq 2$, and $c_{1}=1$. Then,

$$
\begin{aligned}
D_{\sigma}^{k} c^{\varrho}(t, x)= & \sum_{i=1}^{k}\left\langle G\left(t-r_{i}, x-\cdot\right) D_{\hat{\sigma}_{i}}^{k-1} f\left(c^{\varrho}\left(r_{i}, \cdot\right)\right), \varphi_{i}\right\rangle_{\mathcal{H}} \\
& +\int_{\bigvee_{i} r_{i}}^{t} \int_{\mathbb{R}^{d}} G(t-r, x-y) \Delta_{\sigma}\left(f, c^{\varrho}(s, y)\right) \eta(\mathrm{d} s, \mathrm{~d} y) \\
& +\int_{\bigvee_{i} r_{i}}^{t} \int_{\mathbb{R}^{d}} G(t-r, x-y) D_{\sigma}^{k} c^{\varrho}(s, y) f^{\prime}\left(c^{\varrho}(s, y)\right) \eta(\mathrm{d} s, \mathrm{~d} y),
\end{aligned}
$$

where $\Delta_{\sigma}(g, X)=D_{\sigma}^{k} g(X)-g^{\prime}(X) D_{\sigma}^{k} X$.

Now, following similar arguments to the proof of Lemma 3.2 of [12], we can prove that, for any $j \geq 1, k \in \mathbb{N}$, and $p \in[1, \infty)$,

$$
\sup _{0 \leq \varrho \leq 1} \sup _{0 \leq t \leq T} \sup _{x \in \mathbb{R}^{d}}\left\|c_{j}^{\varrho}(t, x)\right\|_{k, p} \leq C .
$$

Then, this bound together with (5.6) and (5.7) implies that

$$
\sup _{0 \leq \varrho \leq 1} \sup _{0 \leq t \leq T} \sup _{x \in \mathbb{R}^{d}}\left\|\hat{c}_{j}^{\varrho}(t, x)\right\|_{k, p} \leq C .
$$

Finally, as in [14], we can check that, for any $t>0$ and $x \in \mathbb{R}^{d}$, there exists a finite and positive constant $C$ such that

$$
\left\|\mid\left\langle D c^{\varrho}(t, x), D c^{\varrho}(t, x)\right\rangle_{\mathscr{H}_{T}}^{-1}\right\|_{p} \leq C \varrho^{-2} \text { for all } p>1, \varrho \in[0,1] .
$$

This bound yields, for some positive constant $C$,

$$
\sup _{0<\varrho \leq 1} \mathrm{E}\left[\left|\left\langle D \hat{c}^{\varrho}(t, x), D \hat{c}^{\varrho}(t, x)\right\rangle^{-1}\right|^{p}\right] \leq C \quad \text { for all } p>1 .
$$

Using the same arguments as in (5.9), we can show that the centered Gaussian variable $c_{1}^{0}(t, x)$ satisfies $\mathrm{E}\left[\left|c_{1}^{0}(t, x)\right|^{2}\right]>0$. 


\subsection{Taylor expansion of the density}

Before giving the main result of this section we briefly present some important notions on Malliavin calculus given in [12], [16], and [17]. Let $X: \Omega \rightarrow \mathbb{R}$ be a Wiener functional and denote its Malliavin matrix by $\Upsilon_{X}$. The random variable $X$ is said to be nondegenerate if $X \in \mathbb{D}^{\infty}$ and $\Upsilon_{X}^{-1} \in \bigcap_{p \geq 1} L^{p}(\Omega)$. Consider nondegenerate variables $X, Y \in \mathbb{D}^{\infty}$ and a smooth function $g$. For any $j \geq 1$, we have the following integral by parts formula:

$$
\mathrm{E}\left[g^{(j)}(X) Y\right]=\mathrm{E}\left[g(X) H_{j}(X, Y)\right],
$$

where $H_{j}(X, Y)$ is a random variable belonging to $\mathbb{D}^{\infty}$ defined recursively as

$$
\begin{gathered}
H_{1}(X, Y)=D^{*}\left(X \Upsilon_{X}^{-1} D Y\right), \\
H_{j}(X, Y)=H_{1}\left(X, H_{j-1}(X, Y)\right), \quad j \geq 2,
\end{gathered}
$$

with $D^{*}$ denoting the Skorokhod integral.

For any $p>1$ and $k, j \in \mathbb{N}$, there exist a positive constant $C(p, k, j)$ and positive real numbers $k_{2}, k_{3}, q_{1}, q_{2}, q_{3}, l_{1}, l_{2}$, and $l_{3}$, depending on $p, k$, and $j$, such that

$$
\left\|H_{j}(X, Y)\right\|_{k, p} \leq C(p, k, j)\left\|\Upsilon_{X}^{-1}\right\|_{q_{1}}^{l_{1}}\|X\|_{k_{2}, q_{2}}^{l_{2}}\|Y\|_{k_{3}, q_{3}}^{l_{3}} .
$$

Finally, the Radon measure defined by $g \rightarrow \mathrm{E}[g(X) Y]$ has a bounded $\mathcal{C}^{\infty}$ density

$$
p(y) \mathrm{E}\left[\mathbf{1}_{\{X>y\}} H_{1}(X, Y)\right] .
$$

Now we can enunciate and prove the main theorem.

Theorem 5.1. Assume that $f$ is $\mathrm{C}^{\infty}$ with bounded derivatives of any order and that there exists an $f_{0}>0$ such that $|f(y)| \geq f_{0}$ for any $y \in \mathbb{R}$. We also suppose that the spectral measure $\mu$ associated to $\varepsilon$ satisfies

$$
\int_{\mathbb{R}^{d}} \frac{\mu(\mathrm{d} \xi)}{\left(1+|\xi|^{2}\right)^{(\alpha+\gamma) q / 2}}<\infty \text { for some } q \in\left(0, \frac{1}{2}\right) .
$$

Let $p_{t, x}^{\varrho}(y)$ be the density of the law of the process $c^{\varrho}(t, x)$. Then, for $(t, x) \in(0, T] \times \mathbb{R}^{d}$ and $\varrho>0$,

$$
p_{t, x}^{\varrho}(0)=\frac{1}{\varrho}\left[\frac{1}{\sqrt{2 \pi \mathrm{E}\left[\left|c_{1}^{0}(t, x)\right|^{2}\right]}}+m_{1} \varrho+\cdots+m_{n} \varrho^{n}+\varrho^{n+1} R_{n+1}(\varrho)\right],
$$

where $c_{1}^{0}(t, x)$ is defined in (5.4). Here the odd coefficients are null and, for even $\ell \geq 2$, we have

$$
m_{\ell}=\frac{1}{\ell !} \mathrm{E}\left[\mathbf{1}_{\left\{c_{1}^{0}(t, x)>0\right\}} M_{\ell}\right],
$$

where

$$
M_{\ell}=\sum^{(1, \ell)} K_{\zeta_{1}, \ldots, \zeta_{k}}^{\ell} H_{k+1}\left(c_{1}^{0}(t, x), \prod_{j=1}^{k} \frac{1}{\varsigma_{j}+1} c_{\varsigma_{j}+1}^{0}(t, x)\right) .
$$

Moreover,

$$
\sup _{0<\varrho \leq 1}\left|R_{n+1}(\varrho)\right|<+\infty
$$


Proof. Let $g$ be a $\mathcal{C}^{\infty}$ function with bounded support included in $\mathbb{R}$. The regularity with respect to $\varrho$ implies that the mapping

$$
\varrho \in(0,1) \rightarrow g\left(\hat{c}^{\varrho}(t, x)\right) \in \mathbb{R}
$$

is $\mathcal{C}^{\infty}$ a.s. Then Taylor's expansion and Leibniz's formula together with (5.6) and (5.7) yield

$$
\begin{aligned}
g\left(\hat{c}^{\varrho}(t, x)\right)= & g\left(c_{1}^{0}(t, x)\right)+\left.\sum_{\ell=1}^{n} \frac{\varrho^{\ell}}{\ell !} \frac{\partial^{\ell}}{\partial \varrho^{\ell}}\left[g\left(\hat{c}^{\varrho}(t, x)\right)\right]\right|_{\varrho=0} \\
& \left.+\varrho^{n+1} \int_{0}^{1} \frac{(1-\tau)^{n}}{n !} \frac{\partial^{n+1}}{\partial \tilde{\tau}^{n+1}}\left[\hat{c}^{\tilde{\tau}}(t, x)\right)\right]\left.\right|_{\tilde{\tau}=\varrho \tau} \mathrm{d} \tau \\
= & g\left(c_{1}^{0}(t, x)\right)+\sum_{\ell=1}^{n} \frac{\varrho^{\ell}}{\ell !} \sum^{(1, \ell)} K_{\beta_{1}, \ldots, \beta_{k}}^{\ell} g^{(k)}\left(c_{1}^{0}(t, x)\right) \prod_{s=1}^{k} \frac{c_{\beta_{s}+1}^{0}(t, x)}{\beta_{s}+1} \\
& +\varrho^{n+1} \int_{0}^{1} \frac{(1-\tau)^{n}}{n !} \sum^{(1, n+1)} K_{\beta_{1}, \ldots, \beta_{k}}^{n+1} g^{(k)}\left(\hat{c}^{\tau \varrho}(t, x)\right) \prod_{s=1}^{k} \hat{c}_{\beta_{s}}^{\tau \varrho}(t, x) \mathrm{d} \tau .
\end{aligned}
$$

Taking expectations and using (5.11) we have

$$
\begin{aligned}
\mathrm{E}\left[g\left(\hat{c}^{\varrho}(t, x)\right)\right]= & \mathrm{E}\left[g\left(c_{1}^{0}(t, x)\right)\right] \\
& +\sum_{\ell=1}^{n} \frac{\varrho^{\ell}}{\ell !} \sum^{(1, \ell)} K_{\beta_{1}, \ldots, \beta_{k}}^{\ell} \mathrm{E}\left[g\left(c_{1}^{0}(t, x)\right) H_{k}\left(c_{1}^{0}(t, x), \prod_{s=1}^{k} \frac{c_{\beta_{s}+1}^{0}(t, x)}{\beta_{s}+1}\right)\right] \\
& +\varrho^{n+1} \int_{0}^{1} \frac{(1-\tau)^{n}}{n !} \sum^{(1, n+1)} K_{\beta_{1}, \ldots, \beta_{k}}^{n+1} \\
& \times \mathrm{E}\left[g\left(\hat{c}^{\tau \varrho}(t, x)\right) H_{k}\left(\hat{c}^{\tau \varrho}(t, x), \prod_{s=1}^{k} \hat{c}_{\beta_{s}}^{\tau \varrho}(t, x)\right)\right] \mathrm{d} \tau .
\end{aligned}
$$

The Radon measure defined by these expectations have $\mathcal{C}^{\infty}$ bounded densities since the random variables $c_{1}^{0}(t, x)$ and $\hat{c}^{\tau \varrho}(t, x)$ are nondegenerate and belong to $\mathbb{D}^{\infty}$ (see (5.8) and (5.10)). Now, using (5.13), we obtain

$$
\hat{p}_{t, x}^{\varrho}(y)=p_{t, x}^{0,1}(y)+\sum_{\ell=1}^{n} \frac{\mathrm{E}\left[\mathbf{1}_{\left\{c_{1}^{0}(t, x)>y\right\}} M(\ell)\right]}{\ell !} \varrho^{\ell}+\varrho^{n+1} R_{n+1}(y, \varrho),
$$

where $\hat{p}_{t, x}^{\varrho}(y)$ and $p_{t, x}^{0,1}(y)$ are the densities of $\hat{c}^{\varrho}(t, x)$ and $c_{1}^{0}(t, x)$, respectively, $M(\ell)$ is given in (5.14) and the remainder $R_{n+1}(y, \varrho)$ is

$$
\int_{0}^{1} \frac{(1-\tau)^{n}}{n !} \mathrm{E}\left[\mathbf{1}_{\left\{\hat{c}^{\tau} \varrho(t, x)>y\right\}} H_{k+1}\left(\hat{c}^{\tau \varrho}(t, x), \sum^{(1, n+1)} K_{\beta_{1}, \ldots, \beta_{k}}^{n+1} \prod_{s=1}^{k} \hat{c}_{\beta_{s}}^{\tau \varrho}(t, x)\right)\right] \mathrm{d} \tau .
$$

With (5.12) in mind, this remainder can be bounded by means of (5.8) and (5.10). Taking account of the facts that

$$
c_{1}^{0}(t, x) \sim \mathcal{N}\left(0, \mathrm{E}\left[\left(c_{1}^{0}(t, x)\right)^{2}\right]\right) \quad \text { and } \quad p_{t, x}^{\varrho}(0)=\frac{1}{\varrho} \hat{p}_{t, x}^{\varrho}(0),
$$

we can conclude the proof of the theorem. 


\section{References}

[1] AdLer, R. J. (1981). The Geometry of Random Fields. John Wiley, Chichester.

[2] Angulo, J. M., Anh, V. V., McVinish, R. And Ruiz-Medina, M. D. (2005). Fractional kinetic equations driven by Gaussian or infinitely divisible noise. Adv. Appl. Prob. 37, 366-392.

[3] Angulo, J. M., Ruiz-Medina, M. D., Anh, V. V. and Grecksch, W. (2000). Fractional diffusion and fractional heat equation. Adv. Appl. Prob. 32, 1077-1099.

[4] Anh, V. V. and Leonenko, N. N. (2001). Spectral analysis of fractional kinetic equations with random data. J. Statist. Phys. 104, 1349-1387.

[5] AnH, V. V. And LeOnenKo, N. N. (2002). Renormalization and homogenization of fractional diffusion equations with random data. Prob. Theory Relat. Fields 124, 381-408.

[6] Anh, V. V., Angulo, J. M. And Ruiz-Medina, M. D. (1999). Possible long-range dependence in fractional random fields. J. Statist. Planning Infer. 80, 95-110.

[7] Dalang, R. C. (1999). Extending the martingale measure stochastic integral with applications to spatially homogeneous s.p.d.e's. Electron. J. Prob. 4, 29pp.

[8] Dalang, R. C. and Frangos, N. E. (1998). The stochastic wave equation in two spatial dimensions. Ann. Prob. 26, 187-212.

[9] Dautray, R. and Lions, J.-L. (1988). Mathematical Analysis and Numerical Methods for Science and Technology, Vol. 2. Springer, Berlin.

[10] Florit, C. ANd Márquez-Carreras, D. (2007). The generalized heat equation. Submitted.

[11] Kunita, H. (1988). Stochastic differential equations and stochastic flow of diffeomorphisms. In École d'Été de Probabilités de Saint-Flour XII-1982 (Lecture Notes Math. 1097), Springer, Berlin, pp. 143-303.

[12] Márquez-Carreras, D. (2006). On the asymptotics of the density in perturbed SPDE's with spatially correlated noise. Infin. Dimens. Anal. Quantum Prob. Relat. Top. 9, 271-285.

[13] Márquez-Carreras, D. and Sanz-Solé, M. (1998). Taylor expansion of the density in a stochastic heat equation. Collect. Math. 49, 399-415.

[14] Márquez-Carreras, D. and Sarrà, M. (2003). Behaviour of the density in perturbed SPDE's with spatially correlated noise. Bull. Sci. Math. 127, 348-367.

[15] Márquez-Carreras, D., Mellouk, M. and Sarrà, M. (2001). On stochastic partial differential equations with spatially correlated noise: smoothness of the law. Stoch. Process. Appl. 93, 269-284.

[16] Nualart, D. (1998). Analysis on Wiener space and anticipating stochastic calculus. In École d'Été de Probabilités de Saint-Flour XXV-1995 (Lecture Notes Math. 1690), Springer, New York, pp. 123-227.

[17] Nualart, D. (1998). Malliavin Calculus and Related Topics. Springer, New York.

[18] Ruiz-Medina, M. D., Angulo, J. M. And Anh, V. V. (2001). Scaling limit solution of a fractional Burgers equation. Stoch. Process. Appl. 93, 285-300.

[19] Samko, S. G., Kilbas, A. A. And Marichev, O. I. (1987). Fractional Integrals and Derivatives. Gordon and Breach, New York.

[20] Schwartz, L. (1966). Théorie des Distributions. Hermann, Paris.

[21] Stein, E. M. (1970). Singular Integrals and Differentiability Properties of Functions. Princeton University Press.

[22] Walsh, J. B. (1986). An introduction to stochastic partial differential equations. In École d'Été de Probabilites de Saint-Flour XIV-1984 (Lecture Notes Math. 1180), Springer, Berlin, pp. 265-439. 